\title{
Interview
}

\section{Brain Observatory and the Continuing Study of H.M.: Interview with Jacopo Annese}

\author{
Rhian Worth ${ }^{\star a}$, Jacopo Annese ${ }^{b}$ \\ Bangor University, Bangor, UK. ${ }^{\mathrm{b}}$ The Brain Observatory, University of California, San Diego, USA.
}

Rhian Worth: What is The Brain Observatory?

Jacopo Annese: The Brain Observatory was established in 2005 when I obtained my first faculty position at UC San Diego (UCSD). The laboratory was originally envisioned as a Hubble telescope for the human brain, in the sense that my long-term goal was to provide public access (for scientists and non-scientists) to views of brain anatomy, the latter created using different neuroimaging modalities including digital microscopy. The name also stems from the fact that many similarities exist, conceptually and technologically, between space exploration and investigation into the complexity of the brain's structural architecture. After all, there is a continuum across the telescope and the microscope; these are two symmetrical instruments, the former probing into the infinitesimally large and the latter resolving the infinitesimally small.

Gradually, The Brain Observatory developed its own brain collection and increased its efforts toward engaging the public in the research that was being conducted in the lab. The fact that we were operating a brain bank and working with real brains actually encouraged participation of local schools in our outreach program. Educational activities are now an integral part of our portfolio.

Rhian Worth: What are the aims of The Brain Observatory - in general terms and in terms of psychology and memory?

Jacopo Annese: The initial goals of The Brain Observatory were technical in nature. We focused on creating the equipment and technological infrastructure to produce maps of the whole human brain at cellular resolution, avoiding the limitations imposed by narrow tissue sampling. There were several reasons for investing start-up funds in protocols that would allow for processing the human brain histologically in one piece, first and foremost the advantage of being able to map neuropathological phenomena across the entire specimen. I was also personally frustrated by the fact that images of neurological disease viewed (or measured) with Magnetic Resonance Imaging 
(MRI) and Diffusion Weighted Imaging (DWI) could not be fully reconciled with follow-up neuropathology reports. This problem plagued the clinical neurosciences since the first mainstream clinical applications of MRI; in fact, while MRI provides low-resolution images of neuropathogenetic phenomena in the whole brain, the traditional postmortem neuropathological report only provides evidence for very few small pieces of tissue. The latter are sampled from one of the hemispheres (with no regard to the handedness of the patient), embedded in paraffin, cut into thin tissue slices, and stained to reveal specific markers of disease, such as neurofibrillary tangles in Alzheimer's disease. The problem is that sampling is very rarely informed by previous MRI and therefore the true extent of damage in the brain could be overlooked.

Another reason for wanting to examine the whole brain postmortem is that there could be distal effects of local lesions that are correlated based on anatomical and functional connectivity. For example, local patches of leucopathology (typically associated with de-myelination) in the deep white matter could produce, by Wallerian degeneration, morphological changes in the cortex according to a very precise pattern of connectivity. Likewise, a high degree of occipital damage in cases of posterior cortical atrophy, a form of dementia that affects primarily visual and visuo-motor functions, could produce effects in the frontal eye fields and the tectum (the superior colliculus).

MRI-neuropathology correlations are an important application of our methodology (Annese, 2012), but the possibility of surveying the whole human brain at the cellular level is also extremely significant if not indispensable to elucidate the neurological basis of human behavior. In particular, where normal cognitive function is perturbed, the study of the brain postmortem has classically complemented neuropsychological tests conducted while the patient was living. This paradigm has been the methodological foundation of single-case brain lesion studies since Paul Broca. The difference is that now we can produce digital histological maps of the brain, combined with 3-Dimensional (3-D) models representing anatomical relationships within the brain to assist follow-up examination of important clinical cases. This ought to greatly facilitate clinico-pathological interpretations of the results of behavioral testing, also because the digital maps are available for web-based remote collaboration.

Moreover, computer-assisted microscopy techniques can also provide quantitative measurements of histo-pathological features associated with cognitive impairment. Potentially, the scores derived from neuropsychological testing can be directly related to MRI-based neuroimaging markers (such as structural volumes).

We know that memory is distributed across a network of grey and white matter structures that include, but are not limited, to the hippocampus,

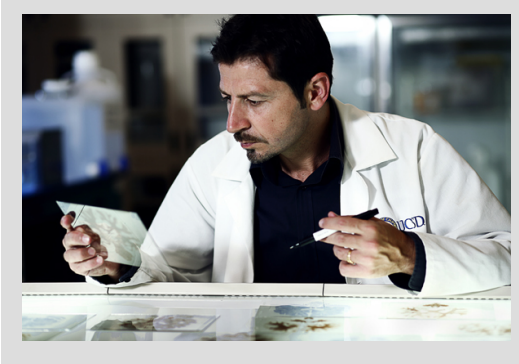

Dr. Jacopo Annese obtained his Bachelor Degree in Biology and Zoology from the University of Rome, "La Sapienza". He completed a Master's Degree in Neurological Science from University College London (London, UK) with Prof. Semir Zeki, and a Ph.D. in Cognitive Neuroscience from Dartmouth College (NH, USA) with Prof. Michael Gazzaniga. Jacopo Annese is director and founder of the Brain Observatory, University of California San Diego. At the Observatory work is under way to develop a public atlas of the human brain and a digital brain library. This innovative project is mostly supported by new technology developed in-house and was featured as the world's most innovative brain bank by the Financial Times (December 2011). In this interview Jacopo discusses research at the Brain Observatory, memory, H.M., and an insight into future of project H.M.

Correspondence: Jacopo Annese, Ph.D., Director, The Brain Observatory, University of California San Diego, 3510 Dunhill Street, CA 92121, USA, Tel: 858-822-4465, Lab: 858-534-3177. Email:jannese@ucsd.edu 
the mammillary bodies, the fimbria, and fornix. Therefore, it is important to apply large-scale digital histology and multimodal analysis to cases where localized damage has produced complex patterns of memory impairment.

Rhian Worth: Researchers at The Brain Observatory are in touch with the donors for a great deal of time before the donor's death - how important is that feature of the research?

Jacopo Annese: Originally, we were working with brain specimens that were obtained from autopsy services linked to hospitals and the Department of Pathology at the UCSD Medical Center. There were two main problems with this mechanism. Firstly, we were seldom able to obtain the whole brain of a patient whose autopsy had been consented by the family or that was prescribed by the hospital. Ordinarily, a brain is examined by a trained pathologist within a few weeks of fixation in formalin. This is a first gross evaluation of the case during which the brain is cut into thick slabs (typically along the coronal plane) and each slab is looked at for signs of gross pathology. Subsequently, several small tissue blocks are then dissected and eventually processed histologically for the neuropathological evaluation. When the brain is donated to a tissue bank, then, as I mentioned earlier, the hemispheres are separated; one hemisphere is frozen fresh for molecular-level research and one is fixed in formalin for classical morphological or pathological studies. Several pieces of brain are shipped to different labs that are involved in studies of diseases or functions that involvespecific brain structures. For example, a laboratory investigating the neuropathologic phenomena associated with Parkinson's disease will request samples from the striatum; i.e., the basal ganglia. The problem is that the tissue that is implicated in the most common neurological conditions (the 'usual suspects' like: basal ganglia, hippocampus, brain stem structures) are quickly extinguished and the rest of the brain remains in the bucket or in the freezer largely unconsidered. Moreover, the results of the research that was conducted in different labs on different 'chunks' of the same brain is not coordinated; researchers at different sites don't know that they are working on the same brain. At any rate, the request of the whole brain for one particular study is generally considered profane.

Therefore, I began working with Organ Procurement Organizations (OPOs) and organizations supporting the teaching of medicine and tissue processing to obtain consent for brain donation directly from the families of the deceased. Essentially, we established the Observatory's own brain bank, which is the backbone of the Digital Brain Library (DBL).

The other limitation with obtaining brain tissue obtained from indirect sources was that very little information was given about the patient or subject (i.e., a neurologically healthy donor) for reasons loosely tied to privacy rules applicable in clinical trials. An autopsy number (indicating the date of death), the cause of death, the age, gender, and condition were the only descriptors that characterized a brain. In most cases, the label on the specimen's container would look something like "A\#245/M/85/AD".

The fact is, in the predominant number of cases the families of those individuals who were enrolled in our brain bank did not wish that the donation remained anonymous. So, we started giving families the opportunity to stay involved in the posthumous work done on the brain. A consent mechanism was created by which the next-of-kin (NOK) or power of attorney of the brain donor could contribute with detailed medical and even biographical information to better characterize the case. That is how we became acquainted with our brains, in some cases discovering that these had belonged to painters, musicians, or mathematicians. Starting a registry of donors who would bequest their brain to research while they are alive was the natural next step. Currently, as soon as a participant enrolls in the DBL project, we conduct a series of MRI scans (if there aren't any restrictions like claustrophobia or the use of a pacemaker) and neuropsychological tests. These are repeated every 6 months or 
once a year. We also administer personality and IQ tests; these supplement structured biographical interviews that are recorded and archived together with the neuroimaging data and scores of cognitive function. Besides the obvious advantage of fully characterizing the brain with multiple individual metrics, the work has also become much more rewarding on a professional and personal level.

Rhian Worth: The Brain Observatory also does some work with the brains of other animals such as dolphins what is the aim or what do you hope to gain from that research? How do you think this might relate or connect to humans?

Jacopo Annese: My background is in zoology, so the interest in animal cognition and comparative anatomy has deep roots. In the past, the Observatory has collaborated with other laboratories on the study of the brain of several species of non-human primates and apes. The U.S. Navy's Marine Mammal Program and Sea World are both based in San Diego; therefore collaborations were built to enhance research on the brain of marine mammals and dolphins in particular. Dolphins are the focus of our comparative branch leveraging on equipment and expertise that was developed to study another equivalently large brain, that of humans. Historically, the brains of cetaceans have been the subject of anatomical investigations seeking the structural determinants of their behavioral specializations, such as echolocation, communication, and even intelligence. The brains of cetaceans (dolphins in particular) show many similarities as well as radical differences in respect to the human brain; understanding how the dolphin brain leverages on typically mammalian, yet uniquely specialized and arranged components, is one of the great challenges of comparative neurobiology. So it is very exciting to be able to do some work in this field.

In regards to memory, dolphins can retain learned skills (procedural memory) and can hold brief representations of events (working memory; Herman, 1980). However, there isn't to-date any clear experimental evidence, (most data was acquired working with bottlenose dolphins, Tursiops truncatus) showing that dolphins can actually remember and act according memories of events. The hippocampus in the dolphin brain is greatly reduced in size, an evolutionary trend that is in stark contrast with the dramatic expansion of the neocortex in this and other cetacean species. Such anatomical specialization may support drastically different methods for long-term information storage and recall. However, it should be noted that dolphins are practically anosmatic, lacking olfactory bulbs and peduncles altogether; so it's quite a riddle.

Another important motivation for advancing this research is that an intense debate is presently underway on whether dolphins should be granted the status of 'persons' on account of their alleged human-like cognitive functioning. The ethical, legal, and socio-economic implications of this debate are substantial, because a resolution would affect whaling, captivity, and the use of dolphins in entertainment. Many of the arguments for and against treating dolphins as 'non-human persons' are based on different interpretations of neuroanatomical evidence; in reality, this evidence is extremely scarce and disorganized. I realized that our lab could contribute to this debate with its neuroimaging and atlasing resources, providing data that might support either argument. I have not yet developed strong views on the dispute above-mentioned; surely, I must concede that a great deal of what we know about the brain and cognition of dolphins stems from studies conducted in captivity. The advantage of the type of work that we conduct at the Observatory is that the validity of scientific information that we create does not depend on theories or hypotheses that may be disproved in the future, so our data will always be valid and useful. Decades from now the elegant histological glass slides that we manufacture may be examined, digitized, and explored by other researchers who will likely benefit from the availability of improved technology and better 
knowledge of brain function. The impartial approach that characterizes the work of an anatomist is also extremely opportune when working on the brains of amnesic patients, because as we know, expert opinions regarding the way human memory works have not yet been harmoniously reconciled into a unified theory, yet.

Rhian Worth: Moving onto project H.M., how did project H.M. come about and how did yourself and The Brain Observatory become involved with H.M.?

Jacopo Annese: In 2007 I wrote a grant application addressed to the National Science Foundation (NSF) that was essentially a 'manifesto' describing a radical approach to the preservation and examination of rare brains that are particularly significant for understanding structure-function relationships. The proposal was meant to support the establishment of the conceptual and methodological foundations for the study of the brain of patient H.M., initials that stand for Henry G. Molaison (Squire, 2009). The initiative was funded via a mechanism that was meant for high-risk, but potentially high-reward projects (the project was also supported partly by the Dana Foundation of New York, a contribution from the ViceChancellor for Health Sciences at UCSD, and private donors). This project at its inception definitely fit the category. The technology for accomplishing the goals of the project had to be built from scratch; also, the fact that patient H.M. was alive at the time meant that the proposal didn't really have a starting date. Accordingly, the administration of the grant was tricky; sufficient funds would also be saved for the actual protocol anytime in the future. Dr. Suzanne Corkin, who at the time was making arrangements with Mr. Molaison's legal power of attorney for the consent for the brain donation, endorsed the plans. And so it goes.

Rhian Worth: What are the aims of project H.M.?

Jacopo Annese: The NSF proposal was almost directly in response to the mishaps that afflicted the study of another iconic brain, that of Albert Einstein. Dr. Thomas Harvey was the pathologist who performed Einstein's autopsy; he fixed the brain and divided it into 240 parcels. Over the years he gave away samples to different researchers until he surrendered the rest of the tissue to Princeton University when he retired. Several studies were conducted in different universities based on the few small samples received; sadly, a comprehensive report on Einstein's brain, one that is informed by modern knowledge, could at this point only be done by piecemealing published accounts of the studies. The most salient findings, published in the journal Lancet (Witelson, Kigar, \& Harvey, 1999), were based on photographs made in 1955 by Dr. Harvey and not on the direct examination of the brain.

Our goal was to create a comprehensive archive containing a collection of anatomical images and histological slides from the brain of H.M., so that the plethora of behavioral and cognitive studies (resulting in over two thousand publications) could be revisited, validated, and interpreted in the light of the actual anatomical and pathological state of the brain. Our prerogative was to provide a model of the brain in its entirety at cellular resolution and a central archive of information that would be available to researchers worldwide. Instead of distributing tissue samples the brain would be 'virtualized' into a centralized imaging resource. The database would be stored in a unique repository that would support independent research probing different levels of complexity within the data.

Rhian Worth: What stage is project H.M. at the moment and what is the next step?

Jacopo Annese: In December 2009, on occasion of the first anniversary of Henry Molaison's death, the brain was cut frozen into 2,401 histological slices during an uninterrupted procedure that lasted fifty-three hours. The 
brain of Henry Molaison is now a collection of finished stained histological slides (about 200) and unprocessed tissue slices that are preserved cryo-genically in a secured freezer. These will remain viable for many years; nevertheless, we are progressively processing new sections also based on the feedback from other researchers. The finished histological slides have been digitized using custom-engineered microscope scanners and have been converted into a format that can be accessed using any web browser at multiple levels of resolution. To-date, the neuroimaging program dedicated to the brain of Henry Molaison generated over ten terabytes of data. These data are extremely diverse, ranging from videos documenting the procedures to terapixel microscopy images and 3-D models derived from MRI and blockface imaging (a tomographic technique that utilizes a digital camera mounted directly over the tissue block). A web site that organizes all the information is under construction and should enable efficient searches as well as ways to contribute to the project with the results of independent analyses and interpretations. This means creating a centralized repository not only for the data, but also for new scientific information that is progressively created by multiple investigators.

We also collected extremely diverse material relative to the life history and neuropsychological profile of Mr. Molaison, as well as (digitized) personal photographs, audio files created from several interviews with psychologists and researchers, and anecdotal accounts from nursing staff and other individuals who met Mr. Molaison. Our current archive also includes research notes, prototype development sketches and models, and formal and informal correspondence with collaborators, producers, and members of the public. The project had enormous resonance in the public and the media. Dozens of news and feature articles were written, as well as TV programs, fictional pieces (several theatrical plays have been produced on the subject of patient H.M., his amnesia, and his brain; one specifically created in collaboration with The Brain Observatory), and web content. The main challenge consists in making all this information accessible at different levels of complexity to accommodate interest from different levels of expertise.

Rhian Worth: The project and the website are very interactive with readers able to ask questions, view videos of the procedure, and very much able to keep up to date with what's going. How important a part is that of The Brain Observatory's work?

Jacopo Annese: Opening up the laboratory to peer review and public curiosity was necessary in my view, because the case was so high-profile and because it was interesting to a very wide and diverse audience. It was important that the radically new protocols that we were about to apply to the study of this brain were discussed beforehand. That is the reason why we shared our plans and documentation of many of our protocols on the web before continuing with the procedures. Irrevocable choices had to be made on the techniques, ranging from processing the specimen in paraffin or frozen, section thickness, and the cryo-protecting solutions for long-term storage of the tissue slices. The protocol was fine-tuned working on other cases, so that eventually there were very few unknowns and only a few major calculated risks. The only truly unpredictable factor in the protocol was the eventuality that the brain would crack during freezing. Having applied the same treatment to several other specimens did not guarantee the safety of the procedure one hundred per cent. Each brain may contain structural weaknesses that contribute to creating the seed regions for cracks during heat transfer when the tissue expands. Judging from the MRI images that were acquired before the brain was prepared for histological processing, H.M. had severe white matter deterioration and lacunae that contained fluid. Nevertheless, the brain had to be frozen to $-40^{\circ} \mathrm{C}$ as quickly as possible to minimize the formation of ice crystals that damaged the tissue, making it unusable for histological analysis. I remember this step as the most 'dramatic', feeling extremely apprehensive as we monitored the temperature of the chilled fluid contained in the freezing chamber. The public did not get a chance to see this 
particular phase of the procedure. The web cameras were turned on after the brain was attached to the microtome stage and cutting had begun. Over the course of three days (from Wednesday, December 2 to Friday, December 4 ) over 400,000 viewers tuned in to watch the cutting procedure. Because the procedure was mostly uninterrupted, we covered every continent's time zones multiple times; thus, the messages that we received via Twitter, Facebook, and our own web site came in in many different languages.

Creating the opportunity for such a level of public engagement in the research on the brain of H.M. was an experiment within the experiment.

Rhian Worth: Is H.M.'s brain the only example of a brain from an individual with a memory disorder at the brain observatory or are there other examples as well?

Jacopo Annese: Before we began the work on patient H.M. we had collaborated with Dr. Larry Squire on the examination of three of his patients, including patient E.P. (Stefanacci, Buffalo, Schmolck, \& Squire, 2000), whose medial temporal lobe lesion, which was much more extensive than that of H.M. was produced by viral encephalitis. One of the main raison d'être for The Digital Brain Library is the possibility of preserving (physically and digitally) the brains of important cases under the same roof and in the same 'cloud' in the form data that can be accessed remotely without restrictions relative to file size and bandwidth. Crucially, all brains are processed exactly the same way, so that direct comparisons can be made across the brains of different patients.

Rhian Worth: What do you think the project will add to research into memory?

Jacopo Annese: This is hard to say at this stage. In my view, the computer-assisted postmortem examination produced two main findings that are likely to stimulate further studies and conjectures. When we examined the medial temporal lobe (MTL) in 3-D, working with a very high-resolution anatomical volume (built from blockface imaging data), it became apparent that a conspicuous portion of the posterior hippocampus was spared because it extended above the line of approach of Scoville's suction tubes. Our length and volume measurements relative to spared hippocampal tissue are higher than those previously estimated from MRI images acquired when patient H.M. was alive (Corkin, Amaral, Gonzalez, Johnson, \& Hyman, 1997; Salat et al., 2006). Furthermore, histologically this tissue does not appear sclerotic or atrophied, despite H.M.'s history of epilepsy and the fact that the entorhinal cortex and a large portion of the fimbria were indeed severed during the operation (no surprise there). It's unlikely that these preliminary observations and the overall project will revolutionize our understanding of memory in the human brain. But I did not have expectations in this regard; I approached the project as an effort of digital preservation and in this respect I am confident that we will contribute to the history of neuroscience in a substantial way. We also hope that this project will introduce new standards in the way that brains of influential neuropsychology patients will be examined and preserved for prospective study. Having said this, the brain of H.M. will be accessible to not only an elite group of scientists, but to the public at large; a student or a non-scientist may very well be discovering the most extraordinary clues contained in the anatomical images, perhaps on the account of the fact that they will be looking where experts don't think is important. There might be surprises in the future.

Rhian Worth: Also included in The Brain Observatory are the brains of 'normal', healthy individuals - what do you think the study of these brains can tell us about the brain and also memory?

Jacopo Annese: Perhaps the rarest anatomical and histological data available to collaborative research is data from normal brains. The terms 'normal', 'normative', or 'control' are relative when it comes to the morphology of 
the brain. We take the stance that there is a continuum of variability of form across the brains of different individuals; so, rather than attempting to establish a 'template' normative brain, we aim at creating the largest possible catalogue of unique brain maps, providing the information necessary to characterize each subject, radiologically and behaviorally. Meaningful patterns of associations between structural and functional parameters can only emerge from the statistical analyses of potentially correlated variables conducted over a large number of cases. Because formal memory tests are also included in the neuropsychological assessment battery (NAB; Stern \& White, 2003) that we perform with our participants (tests include verbal explicit learning, visual explicit learning, verbal delayed free recall, and visual delayed recognition memory), at some point it will be possible to evaluate the correlation between specific memory functions and different morphological features. Because the brain will eventually be examined microscopically, these features will range from MRI-based measures of cortical thickness of subcortical volumes to estimates of neuronal number and size in specific compartments of the hippocampus and related MTL structures.

Rhian Worth: What is the future of project H.M. and its research and The Brain Observatory both in general and in what the project might be able to tell us about memory?

Jacopo Annese: The ultimate goal of project H.M. is to make all data transparent to the widest and most diverse utilization, and enable multiple representations of the extraordinary archive that preserves Henry Molaison as a person, a brain, and a project. The project will very likely acquire a life of its own. The outcomes are not easily predictable; but I am sure it will be successful in creating brand new connections between very different communities and fields. This is also the overall mission of The Brain Observatory where ideally the boundaries between (neuro) science, the arts, public engagement and education would eventually disappear. The Digital Brain Library embodies these concepts; in fact, the 'gallery' of brains will contain more than just neurological and neuroimaging data. My goal in the next few years is to provide a comprehensive neuroimaging and digital curation service for brains that may have been already collected but that are deteriorating in their current storage conditions or cases that are still being studied by neuropsychologists or neurologists and who deserve being immortalized via an attentive and compassionate postmortem preservation plan.

Web Links:

http://thebrainobservatory.ucsd.edu

\section{References}

Annese, J. (2012). The importance of combining MRI and large-scale digital histology in neuroimaging studies of brain connectivity and disease. Frontiers in Neuroinformatics, 6, , Article 13. doi:. doi:10.3389/fninf.2012.00013

Corkin, S., Amaral, D. G., Gonzalez, R. G., Johnson, K. A., \& Hyman, B. T. (1997). H. M.'s medial temporal lobe lesion: Findings from magnetic resonanceimaging. The Journal of Neuroscience, 17, 3964-3979.

Herman, L. M. (1980). Cognitive characteristics of dolphins. In L. M. Herman (Ed.), Cetacean behavior: Mechanisms and function (pp. 363-429). New York: Wiley-Interscience.

Salat, D. H., van der Kouwe, A. J. W., Tuch, D. S., Quinn, B. T., Fischl, B., Dale, A. M., \& Corkin, S. (2006). Neuroimaging H.M.: A 10-year follow-up examination. Hippocampus, 16(11), 936-945. doi:10.1002/hipo.20222

Squire, L. R. (2009). The Legacy of Patient H.M. for Neuroscience. Neuron, 61(1), 6-9. doi:10.1016/j.neuron.2008.12.023 
Stefanacci, L., Buffalo, E., Schmolck, H., \& Squire, L. R. (2000). Profound amnesia after damage to the medial temporal lobe: A neuroanatomical and neuropsychological profile of patient E. P. The Journal of Neuroscience, 20(18), 7024-7036.

Stern, R. A., \& White, T. (2003). Neuropsychological Assessment Battery. Lutz, FL: Psychological Assessment Resources, Inc.

Witelson, S. F., Kigar, D. L., \& Harvey, T. (1999). The exceptional brain of Albert Einstein. Lancet, 353, 2149-2153. doi:10.1016/S0140-6736(98)10327-6 